\title{
Physical studies of asteroids
}

\section{The spin rate of M-type asteroids ${ }^{\star}$}

\author{
C.-I. Lagerkvist ${ }^{1}$, I. Belskaya ${ }^{2}$, A. Erikson ${ }^{3}$, V. Schevchenko ${ }^{2}$, S. Mottola $^{3}$, V. Chiorny ${ }^{2}$, P. Magnusson ${ }^{1}$, \\ A. Nathues ${ }^{3}$, and J. Piironen ${ }^{4}$ \\ 1 Astronomiska Observatoriet, Box 515, S-752 20 Uppsala, Sverige \\ 2 Astronomical Observatory, 35 Sumskaya Str., 310022 Kharkiv, Ukraine \\ 3 Institute of Planetary Exploration, DLR, Rudower Chaussee 5, 12489 Berlin, Germany \\ ${ }^{4}$ Observatory, P.O. Box 14, FIN-00014, University of Helsinki, Finland
}

Received December 12, 1997; accepted January 30, 1998

\begin{abstract}
The results from photometric lightcurve observations of nine M-type asteroids are presented. New rotation periods were determined for 6 asteroids: 217 Eudora (12.54 h), 322 Phaeo (17.56 h), 572 Rebekka (5.65 h), 757 Portland $(6.58 \mathrm{~h}), 857$ Glasenappia $(8.23 \mathrm{~h})$ and 872 Holda $(7.20 \mathrm{~h}) . B-V$ colour measurements of seventeen previously unclassified asteroids add seven asteroids to the known M-type population.

An excess of fast rotators among M-type asteroids compared to asteroids of other taxonomic types is evident. The six asteroids with slow spin rates, but hitherto classified as M, are shown to have classification parameters untypical for the M-type population.
\end{abstract}

Key words: asteroids

\section{Introduction}

The present paper is a continuation of our study of Mtype asteroids (Belskaya \& Lagerkvist 1996). The purpose is to enlarge the data set of rotation periods of asteroids of taxonomic type M. Previous studies of the spin rates of M-type asteroids have shown, with varying statistical significance, that on average M-type asteroids have faster spin than asteroids of other taxonomic types (Harris \& Burns 1979; Dermott \& Murray 1982; Lagerkvist 1983; Lagerkvist et al. 1985; Binzel et al. 1989; Belskaya \& Lagerkvist 1996).

There are two main problems in analysing the distribution of spin rates for M-type objects. One is the small

Send offprint requests to: C.-I. Lagerkvist

* Partly based on observations collected at the European Southern Observatory, La Silla, Chile. number of M-type asteroids with known rotation periods. In this paper we present observations of nine M-type asteroids, resulting in new determinations of spin rates for six of these. This gives an increase of more than $15 \%$ of the known spin rates of asteroids of taxonomic type M. In order to increase the known population of M-type asteroids we have determined $B-V$ colour indices for 17 asteroids with moderate albedos. The second problem is the inconsistent classification of some asteroids between different taxonomies. Below we discuss the reliability of the classification for some peculiar objects.

\section{Observations and results}

\subsection{Photometry}

The photometric observations were made in 1984-1996 at four different sites within the frame of a joint observational program. We used the following telescopes: 0.6 $\mathrm{m}$ Swedish telescope at La Palma (LP), Canary Islands, $61 \mathrm{~cm}$ Bochum, 50-cm and $1 \mathrm{~m}$ ESO telescopes at ESO, La Silla, Chile and the 70-cm telescope of Astronomical Observatory of Kharkiv State University (KhAO). The observations at $\mathrm{KhAO}$ and with the Bochum telescope were CCD observations, otherwise conventional photoelectric photometry was used. All the photometric reductions were made with standard methods. The data are corrected for light-time. Table 1 contains the aspect data of the observed asteroids and references to the used telescopes. The obtained rotation periods and amplitudes are given in Table 2. The errors in the rotation periods are indicated by the number of decimals given. The amplitude errors are normally of a few hundreds of a magnitude. Figures 1-11 present the composite lightcurves of individual asteroids. Below we discuss each asteroid in more detail. 
Table 1. Aspect data

\begin{tabular}{|c|c|c|c|c|c|c|c|}
\hline Asteroid & $\begin{array}{l}\text { Date } \\
\text { (UT) }\end{array}$ & $\begin{array}{c}r \\
(\mathrm{AU})\end{array}$ & $\begin{array}{c}\Delta \\
(\mathrm{AU})\end{array}$ & $\begin{array}{r}\alpha \\
\left({ }^{\circ}\right)\end{array}$ & $\begin{array}{r}\lambda_{2000} \\
\left({ }^{\circ}\right)\end{array}$ & $\begin{array}{r}\beta_{2000} \\
\left({ }^{\circ}\right)\end{array}$ & Telescope \\
\hline 97 Klotho & 1995 Mar.-26.3 & 3.149 & 2.313 & 11.6 & 222.8 & 12.6 & Bochum $61 \mathrm{~cm}$ \\
\hline 97 Klotho & 1995 Mar.-27.3 & 3.151 & 2.306 & 11.3 & 222.7 & 12.7 & Bochum $61 \mathrm{~cm}$ \\
\hline 97 Klotho & 1995 Apr.-29.3 & 3.201 & 2.217 & 4.5 & 216.0 & 14.3 & Bochum $61 \mathrm{~cm}$ \\
\hline 97 Klotho & 1995 May-4.1 & 3.208 & 2.229 & 5.2 & 214.9 & 14.4 & Bochum $61 \mathrm{~cm}$ \\
\hline 97 Klotho & 1995 May-5.1 & 3.210 & 2.232 & 5.3 & 214.7 & 14.4 & Bochum $61 \mathrm{~cm}$ \\
\hline 217 Eudora & 1996 Apr.-25.9 & 2.799 & 1.810 & 4.7 & 212.1 & 12.6 & KhAO $70 \mathrm{~cm}$ \\
\hline 217 Eudora & 1996 May-25.8 & 2.702 & 1.851 & 14.1 & 206.1 & 12.9 & $\mathrm{KhAO} 70 \mathrm{~cm}$ \\
\hline 217 Eudora & 1996 May-26.8 & 2.699 & 1.856 & 14.4 & 205.9 & 12.9 & KhAO $70 \mathrm{~cm}$ \\
\hline 322 Phaeo & 1996 Apr.-16.0 & 3.405 & 2.408 & 2.4 & 206.4 & -8.3 & Bochum $61 \mathrm{~cm}$ \\
\hline 322 Phaeo & 1996 Apr.-17.0 & 3.404 & 2.407 & 2.5 & 206.2 & -8.3 & Bochum $61 \mathrm{~cm}$ \\
\hline 322 Phaeo & 1996 Apr.-21.0 & 3.400 & 2.406 & 3.0 & 205.4 & -8.2 & Bochum $61 \mathrm{~cm}$ \\
\hline 322 Phaeo & 1996 Apr.-23.0 & 3.398 & 2.407 & 3.4 & 204.9 & -8.1 & Bochum $61 \mathrm{~cm}$ \\
\hline 337 Devosa & 1995 Sep.-20.2 & 2.323 & 1.345 & 7.4 & 14.0 & 2.6 & ESO $50 \mathrm{~cm}$ \\
\hline 558 Carmen & 1992 Sep.-3.3 & 2.889 & 2.099 & 14.6 & 26.9 & -8.6 & ESO $1 \mathrm{~m}$ \\
\hline 558 Carmen & 1992 Sep.-5.3 & 2.888 & 2.080 & 14.1 & 26.7 & -8.7 & ESO $1 \mathrm{~m}$ \\
\hline 572 Rebekka & 1996 Feb.-17.1 & 2.479 & 1.533 & 8.4 & 131.6 & -14.4 & Bochum $61 \mathrm{~cm}$ \\
\hline 572 Rebekka & 1996 Feb.-18.1 & 2.481 & 1.538 & 8.7 & 131.4 & -14.3 & Bochum $61 \mathrm{~cm}$ \\
\hline 572 Rebekka & 1996 Feb.-20.2 & 2.484 & 1.550 & 9.4 & 130.9 & -14.2 & Bochum $61 \mathrm{~cm}$ \\
\hline 757 Portlandia & 1996 Sep.-29.9 & 2.135 & 1.439 & 23.7 & 66.0 & 4.1 & KhAO $70 \mathrm{~cm}$ \\
\hline 757 Portlandia & 1996 Sep.-30.9 & 2.134 & 1.429 & 23.5 & 66.1 & 4.2 & KhAO $70 \mathrm{~cm}$ \\
\hline 757 Portlandia & 1996 Oct.-9.9 & 2.130 & 1.344 & 21.0 & 66.5 & 5.1 & KhAO $70 \mathrm{~cm}$ \\
\hline 757 Portlandia & 1996 Nov.-12.9 & 2.119 & 1.145 & 6.6 & 62.2 & 8.6 & $\mathrm{KhAO} 70 \mathrm{~cm}$ \\
\hline 757 Portlandia & 1996 Nov.-13.9 & 2.118 & 1.143 & 6.2 & 61.9 & 8.7 & $\mathrm{KhAO} 70 \mathrm{~cm}$ \\
\hline 857 Glasenappia & 1997 May-6.0 & 2.251 & 1.317 & 12.8 & 196.1 & 7.3 & KhAO $70 \mathrm{~cm}$ \\
\hline 857 Glasenappia & 1997 May-7.0 & 2.250 & 1.322 & 13.2 & 195.9 & 7.3 & $\mathrm{KhAO} 70 \mathrm{~cm}$ \\
\hline 857 Glasenappia & 1997 May-8.0 & 2.249 & 1.326 & 13.6 & 195.7 & 7.2 & KhAO $70 \mathrm{~cm}$ \\
\hline 872 Holda & 1995 Oct.-28.0 & 2.931 & 2.071 & 11.6 & 357.9 & 1.0 & Bochum $61 \mathrm{~cm}$ \\
\hline 872 Holda & 1995 Oct.-30.0 & 2.932 & 2.089 & 12.2 & 357.7 & 1.0 & Bochum $61 \mathrm{~cm}$ \\
\hline 872 Holda & 1996 Nov.-14.9 & 2.866 & 2.065 & 13.7 & 95.4 & -9.4 & KhAO $70 \mathrm{~cm}$ \\
\hline 872 Holda & 1996 Dec.-18.9 & 2.843 & 1.871 & 3.8 & 89.3 & -10.8 & KhAO $70 \mathrm{~cm}$ \\
\hline
\end{tabular}

\section{Klotho:}

This asteroid has previously been observed during six apparitions. Harris \& Young (1983) determined a rotation period of 35 hours and pointed out that this was the longest period among the known M-type objects. Lagerkvist et al. (1988) determined a rotation period of 35.58 hours. Data obtained by Dotto et al. (1992) agree with this period but they do not cover a whole rotation cycle. The extensive observational run undertaken by Lagerkvist et al. (1995) confirmed the slow rotation (35.0 hours) of 97 Klotho. Our observations during three apparitions also give a long rotation period. The observations in 1995, made during March-May, gave us a possibility to define a more precise value of the rotation period. A period of 35.15 hours is the best solution from our observations (Fig. 1). All previously obtained data of 97 Klotho agree well with this rotation period.

\section{Eudora:}

This asteroid was classified to be of type M by Belskaya et al. (1991) based on its colour indices and the value of

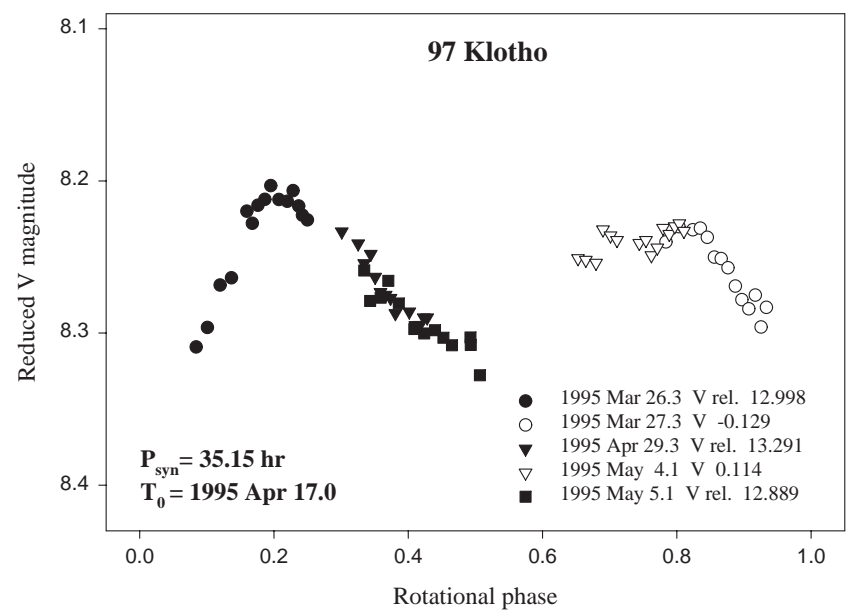

Fig. 1. Composite lightcurve of 97 Klotho

minimum polarization $(0.82 \%)$, which are typical for Mtype objects. The IRAS albedo (0.05), however, is more typical for P-type asteroids. Since the agreement between polarimetric and radiometric albedos is poor we included 


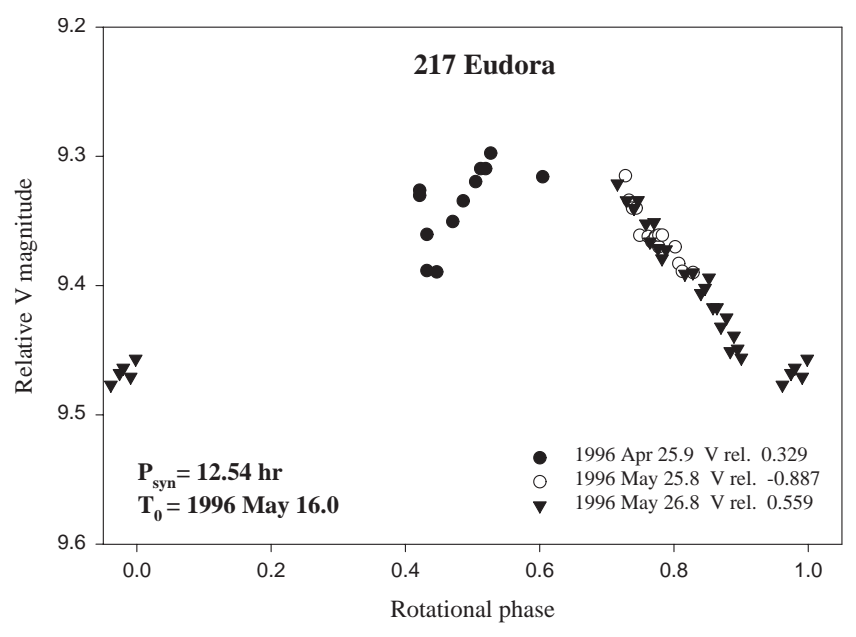

Fig. 2. Composite lightcurve of 217 Eudora

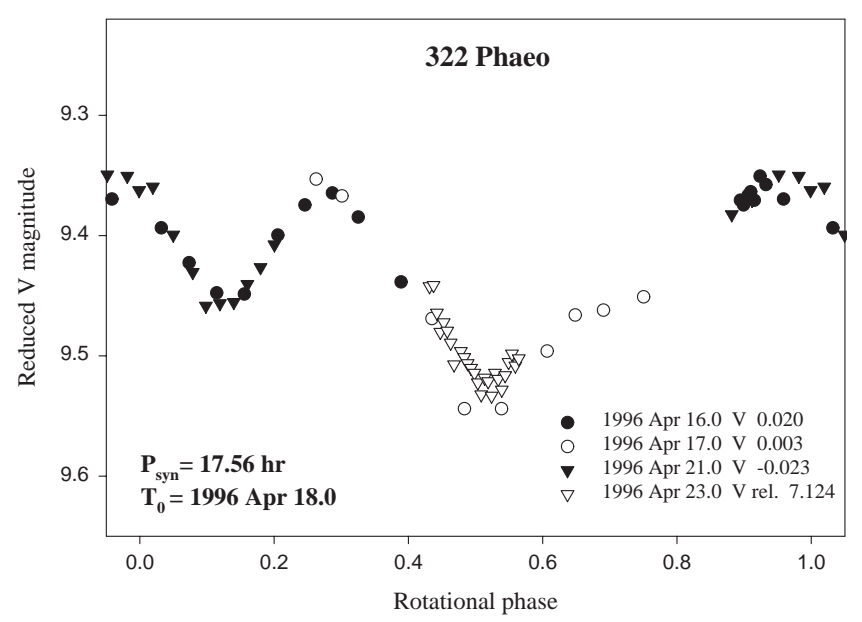

Fig. 3. Composite lightcurve of 322 Phaeo

the asteroid in the list of possible M-type objects. From our observations during three nights we estimate a rotation period of 12.54 hours (Fig. 2) as the most probable one assuming a standard lightcurve with two pairs of extrema. Unfortunately, the obtained data do not give a possibility to determine the rotation period uniquely.

\section{Phaeo:}

Harris \& Young (1983) observed 322 Phaeo but only during a short interval of time. They found only minor changes of the magnitude and concluded that the rotation period was long. Our observations during four nights gave an amplitude of about $0.2 \mathrm{mag}$ and an unambiguous rotation period of 17.56 hours (Fig. 3 ).

\section{Devosa:}

This asteroid has previously been observed during eight apparitions (see Lagerkvist et al. 1996, for detailed references). Lightcurves of 337 Devosa show an asymmetrical shape with three pairs of extrema. We observed 337 Devosa in September 1995 during more than a complete rotational cycle in the $B$ and $V$ bands. The lightcurve

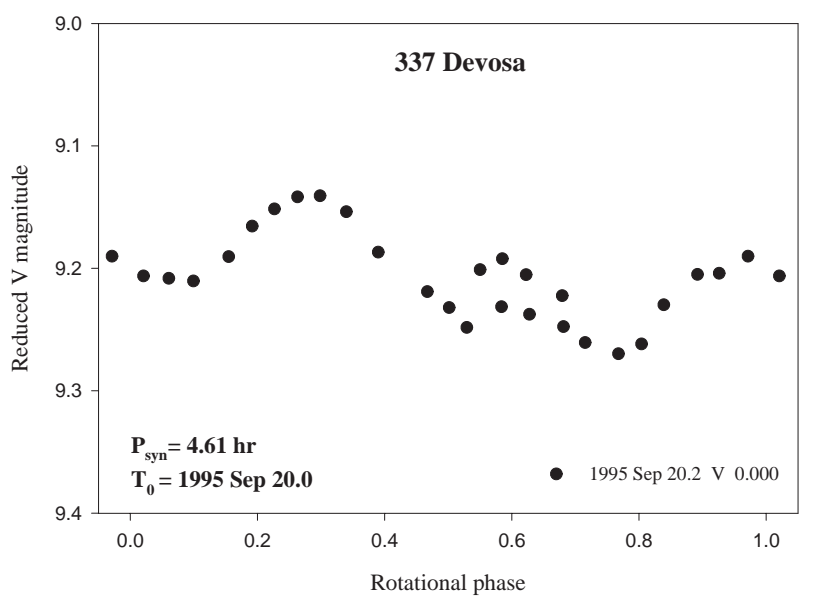

Fig. 4. Composite lightcurve of 337 Devosa

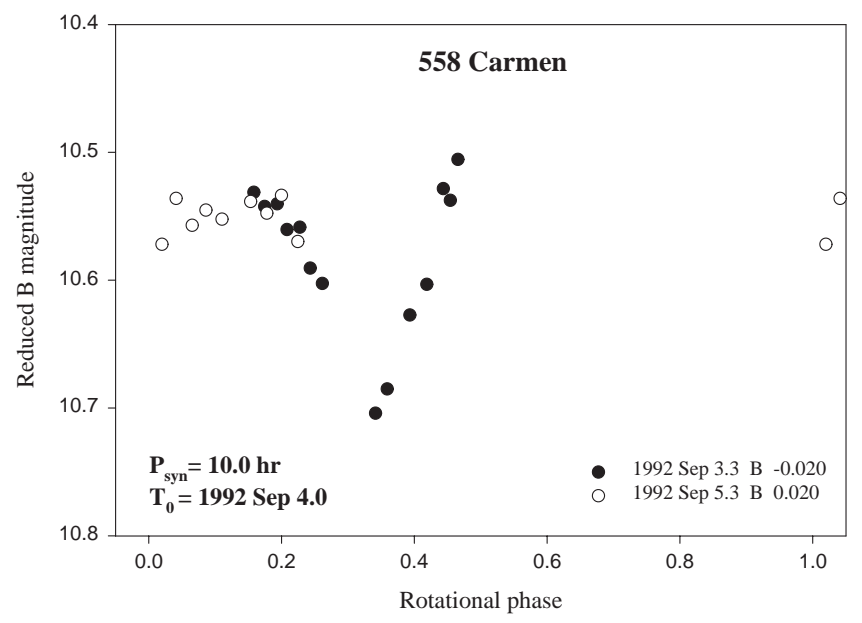

Fig. 5. Composite lightcurve of 558 Carmen

in the $V$ band is shown in Fig. 4. The scatter around rotational phase 0.6 was caused by varying photometric conditions during the night.

\section{Carmen:}

The asteroid 558 Carmen was previously observed during three apparitions by Harris \& Young $(1979,1989)$ and Harris et al. (1992) who estimated the period to 10 hours. Our observations during two nights agree with this period (Fig. 5).

\section{Rebekka:}

Our observations during three nights give a rotation period of 5.65 hours. The composite lightcurve is quite normal with two pairs of extrema and the lightcurve amplitude is $0.3 \mathrm{mag}$ (Fig. 6).

\section{Portlandia:}

From our observations during five nights we determined an unambiguous rotation period of 6.58 hours. The lightcurve is irregular and its shape changed noticebly as the phase angle decreased (Figs. 7 and 8). 


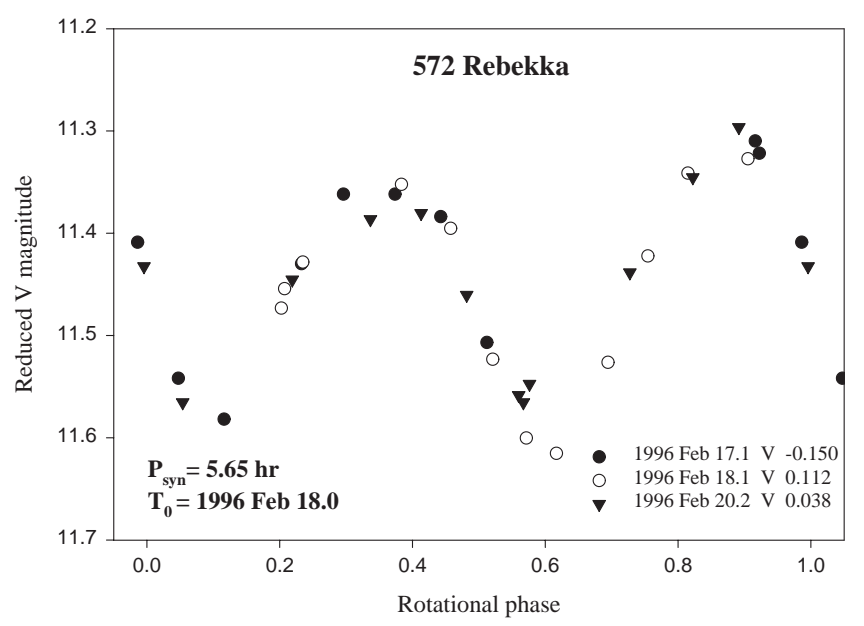

Fig. 6. Composite lightcurve of 572 Rebekka

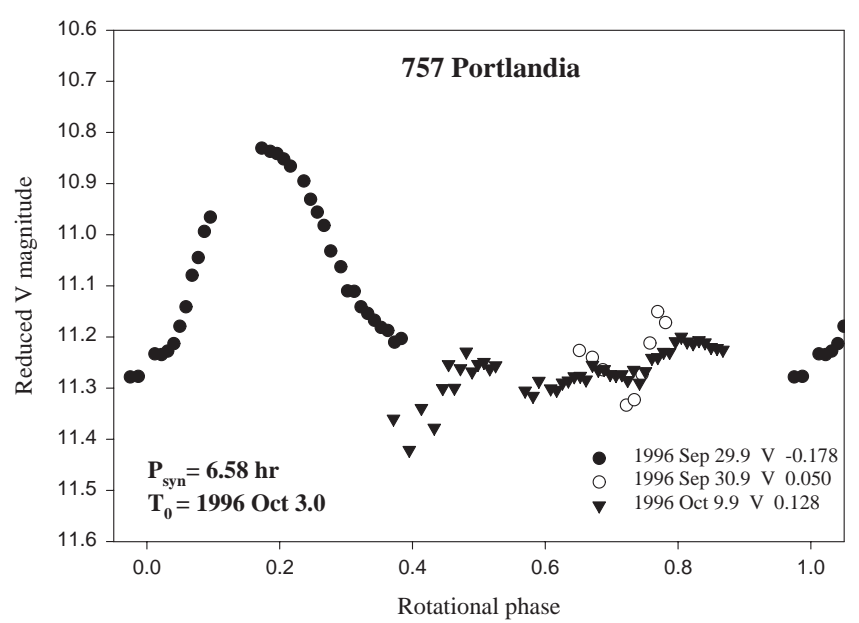

Fig. 7. Composite lightcurve of 757 Portlandia. The phase angle is high, $21^{\circ}-23^{\circ}$

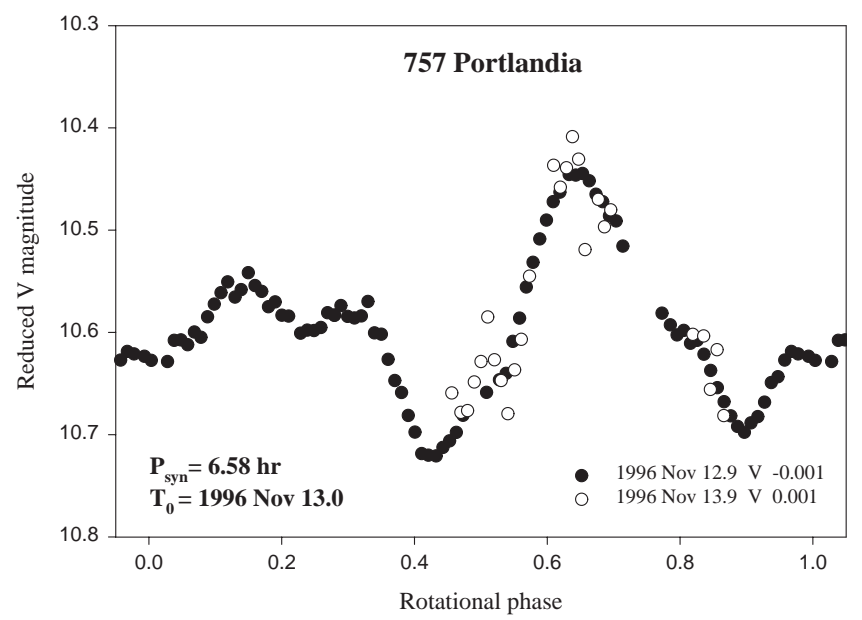

Fig. 8. Composite lightcurve of 757 Portlandia obtained at much lower phase angles than in Fig. $7\left(6^{\circ}-7^{\circ}\right)$

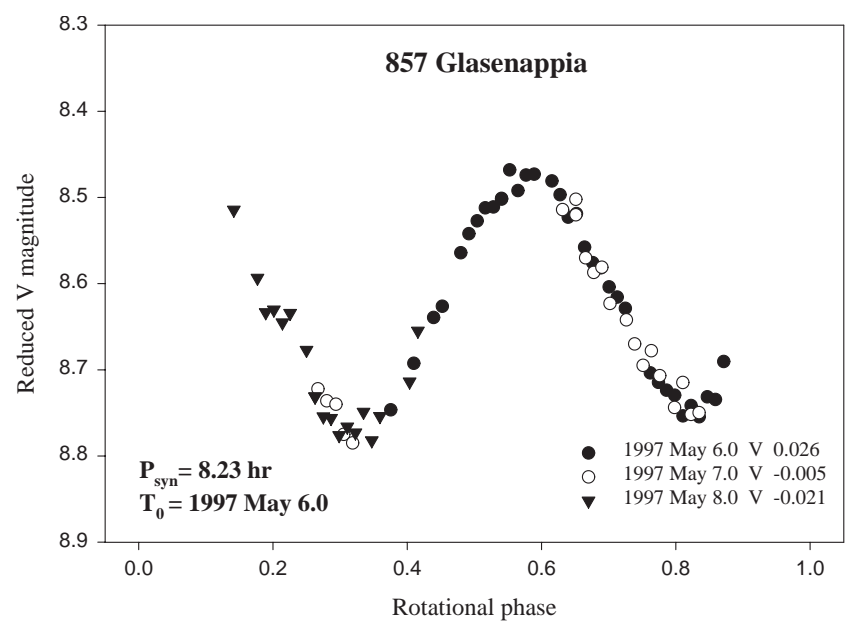

Fig. 9. Composite lightcurve of 857 Glasenappia

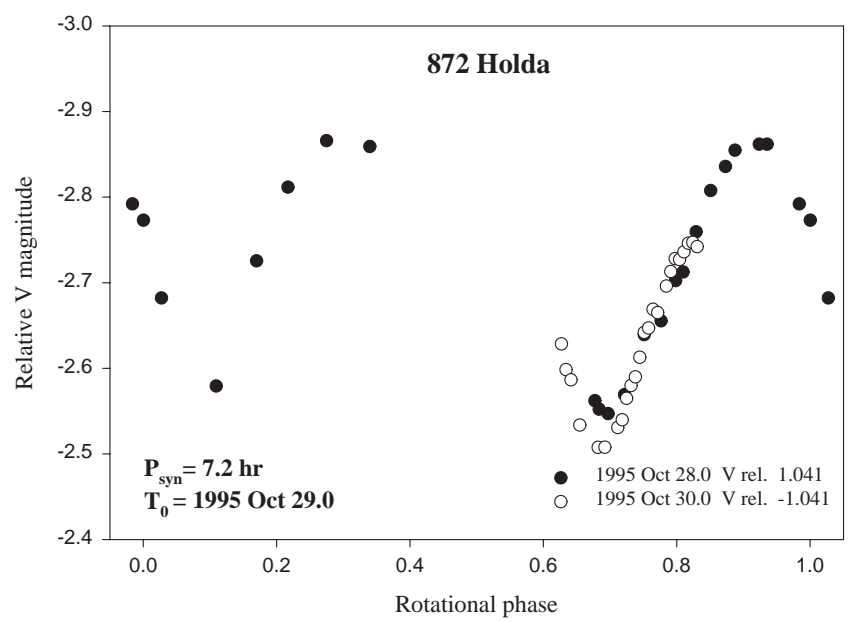

Fig. 10. Composite lightcurve of 872 Holda

\section{Glasenappia:}

Our observations during three nights give a rotation period of 8.23 hours and a lightcurve amplitude of 0.35 mag. The composite lightcurve shown in Figure 9 is regular with two pairs of extrema.

\section{Holda:}

We observed 872 Holda during two apparitions, each time during two nights. The obtained lightcurves are rather regular with amplitudes of $0.2-0.4$ mag depending on the aspect angle. Two possible values of the rotation period were determined: 6.78 or 7.20 hours. Composite lightcurves based on a period of 7.20 hours are shown in Figs. 10 and 11. More observations are needed to define an unambiguous value of the rotation period.

\subsection{Colour indices of selected asteroids}

In order to increase the sample of known M-type asteroids we observed 17 previously unclassified asteroids for which good-quality IRAS albedos were known. For our purpose 


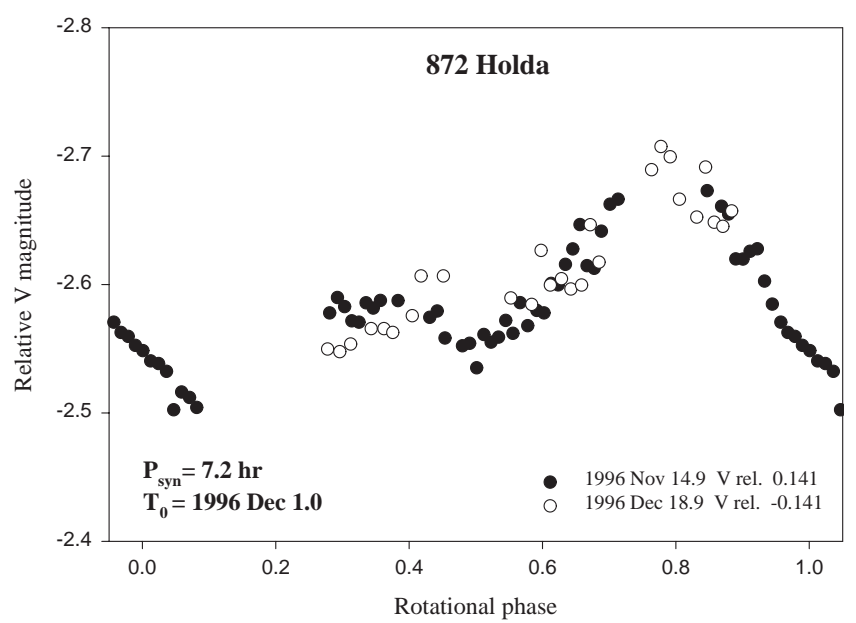

Fig. 11. Composite lightcurve of 872 Holda

Table 2. Rotational periods and lightcurve amplitudes

\begin{tabular}{clrr}
\hline \multicolumn{1}{c}{ Asteroid } & \multicolumn{2}{c}{ Rotation period } & Amplitude \\
& old & new & \\
\hline 97 Klotho & 35.58 & 35.15 & 0.14 \\
217 Eudora & - & 12.54 & 0.16 \\
322 Phaeo & - & 17.56 & 0.20 \\
337 Devosa & 4.61 & confirmed & 0.13 \\
558 Carmen & 10.0 & confirmed & $>0.20$ \\
572 Rebekka & - & 5.65 & 0.30 \\
757 Portland & - & 6.58 & 0.45 \\
857 Glasenappia & - & 8.23 & 0.35 \\
872 Holda & - & 7 & 0.34 \\
\hline
\end{tabular}

we chose asteroids with moderate albedo which are wellseparated into the taxonomic types S, M, A and V according to their $B-V$ colour index (Zellner \& Bowell 1979). The observations were carried out in the standard $B$ and $V$ bands using the 1-m telescope at ESO, Chile in 1993 and the $61 \mathrm{~cm}$ Bochum telescope at ESO during 1994. During each night a set of standard stars were carefully observed. Table 3 gives the asteroid number and name, the measured magnitude in the $V$ band, the $B-V$ colour index, albedo and diameter according to IRAS data (Tedesco et al. 1992) In the last column we give our classification of these asteroids. Seven asteroids have $B-V$ colours within the range of the M-type population, eight asteroids with the S-type and one asteroid have a $B-V$ colour at the border of the $\mathrm{M}$ and $\mathrm{S}$ type populations. The asteroid 1562 Gondolatch is characterized by an extremely large $B-V=1.04 \mathrm{mag}$ which indicates that it is of type A. At size ranges larger than $50 \mathrm{~km}$ the ratio between $\mathrm{S}$ and M-type asteroids is close to three (Zellner \& Bowell 1979). However, in our sample, which is mainly composed of objects smaller than $50 \mathrm{~km}$, this ratio is two. We do not want to draw too firm conclusions from the statistics with small numbers but there is a possibility that for smaller asteroids the proportion of $\mathrm{M}$ type asteroids is higher than for larger objects.

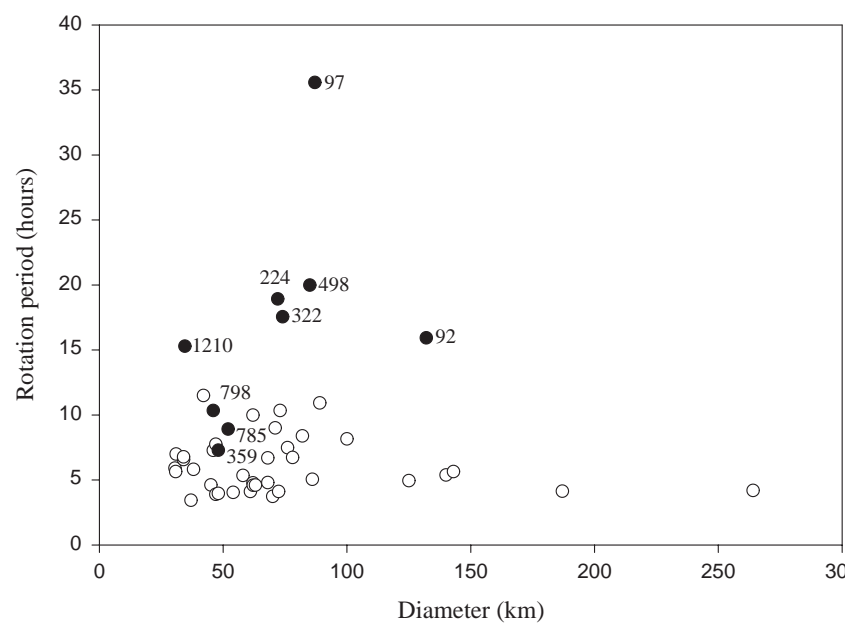

Fig. 12. Rotation period plotted versus diameter for M-type asteroids
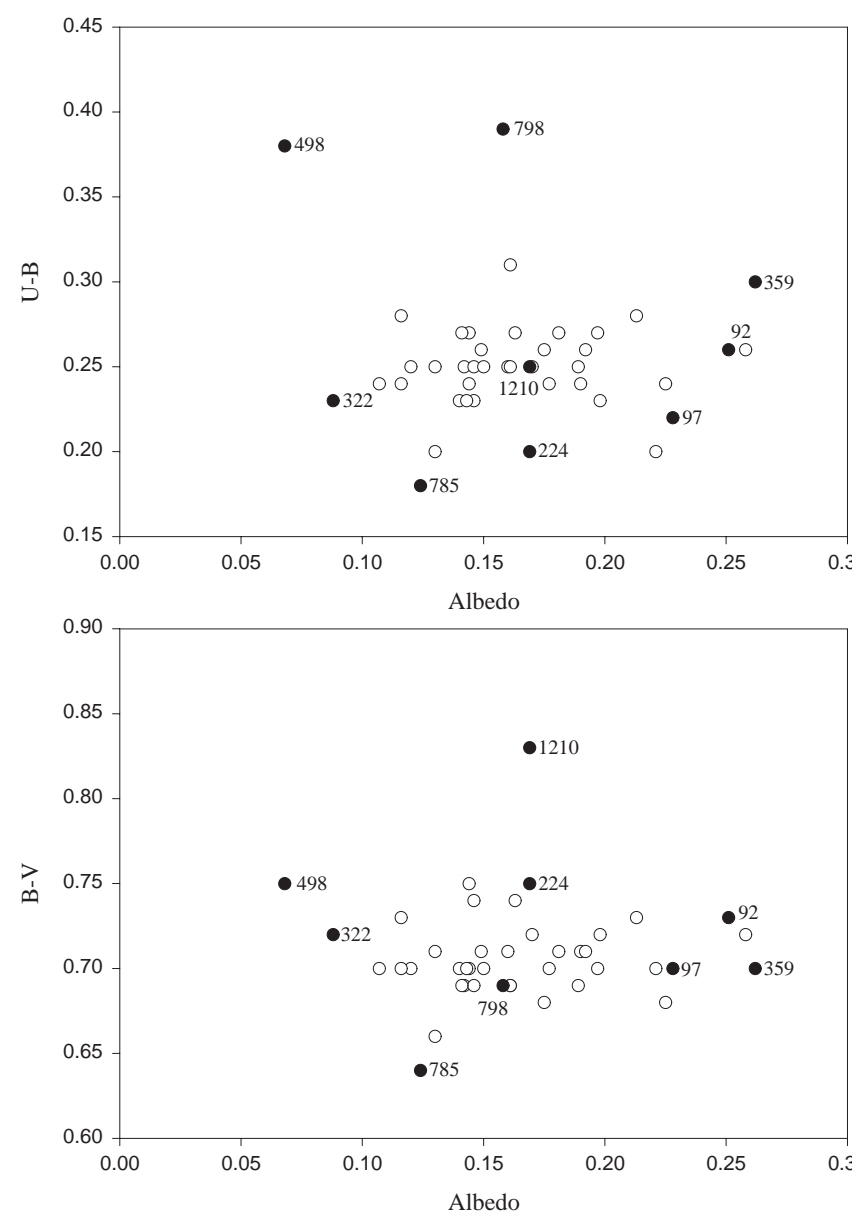

Fig. 13. $U-B$ and $B-V$ versus albedo for $M$ type asteroids 
Table 3. Results of $B-V$ measurements

\begin{tabular}{llrrrr}
\hline Asteroid & $V$ & $B-V$ & Alb. & $\begin{array}{r}\text { Diam. } \\
(\mathrm{km})\end{array}$ & Type \\
\hline 278 Paulina & 12.60 & 0.95 & 0.25 & 35 & $\mathrm{~S}$ \\
427 Galene & 15.20 & 0.76 & 0.24 & 30 & $\mathrm{MS}$ \\
485 Genua & 12.51 & 0.86 & 0.21 & 64 & $\mathrm{~S}$ \\
500 Selinur & 13.80 & 0.82 & 0.17 & 44 & $\mathrm{~S}$ \\
507 Laodica & 15.45 & 0.65 & 0.21 & 44 & $\mathrm{M}$ \\
543 Charlotte & 14.92 & 0.66 & 0.26 & 34 & $\mathrm{M}$ \\
597 Bandusia & 14.05 & 0.88 & 0.24 & 36 & $\mathrm{~S}$ \\
700 Auravictrix & 13.74 & 0.90 & 0.25 & 15 & $\mathrm{~S}$ \\
715 Transvaalia & 15.60 & 0.70 & 0.26 & 29 & $\mathrm{M}$ \\
862 Franzia & 14.71 & 0.83 & 0.14 & 27 & $\mathrm{~S}$ \\
986 Amelia & 13.26 & 0.81 & 0.12 & 51 & $\mathrm{~S}$ \\
1098 Hahone & 13.93 & 0.70 & 0.24 & 25 & $\mathrm{M}$ \\
1334 Lundmarka & 14.24 & 0.65 & 0.18 & 31 & $\mathrm{M}$ \\
1562 Gondolatsch & 14.58 & 1.04 & 0.25 & 11 & $\mathrm{~A}$ \\
2237 Melnikov & 15.23 & 0.72 & 0.13 & 20 & $\mathrm{M}$ \\
3036 Krat & 14.90 & 0.68 & 0.12 & 42 & $\mathrm{M}$ \\
3259 1984 SZ4 & 13.89 & 0.97 & 0.10 & 38 & $\mathrm{~S}$ \\
\hline
\end{tabular}

\section{Discussion}

\subsection{Slowly spinning $M$ asteroids}

A list of M-type asteroids consisting of 60 objects was given by Belskaya \& Lagerkvist (1996). It included all asteroids which were classified as $\mathrm{M}$ at least in one of the available taxonomic systems. At present rotation periods are known for 49 asteroids in this list. All classified asteroids with diameters larger than $50 \mathrm{~km}$ have their rotation rates determined. However, there are still some unclassified objects in this size range. The statistics is practically complete for M-asteroids with diameters larger than 70 km (Belskaya \& Lagerkvist 1996).

The rotation periods versus diameters are shown in Fig. 12 for M-type asteroids. There are six asteroids having a rotation period longer than 15 hours (black dots). The other three black dots in the figure represents asteroids with colour indices or albedos differing from typical Mtype values.

How typical are the classification parameters for these asteroids? To answer this question we have plotted in Fig. $13 U-B$ and $B-V$ colour indices versus albedo for the known M-type asteroids. These parameters are critical for distinguishing M-type objects from others. One can see that many slow rotators have albedos and (or) colour indices different from the typical values for the M-type asteroids. Also the asteroids 359, 785 and 798 have classification parameters that are non-typical for asteroids of type M. Below we give more detailed comments on the six M-type asteroids with long rotation periods.
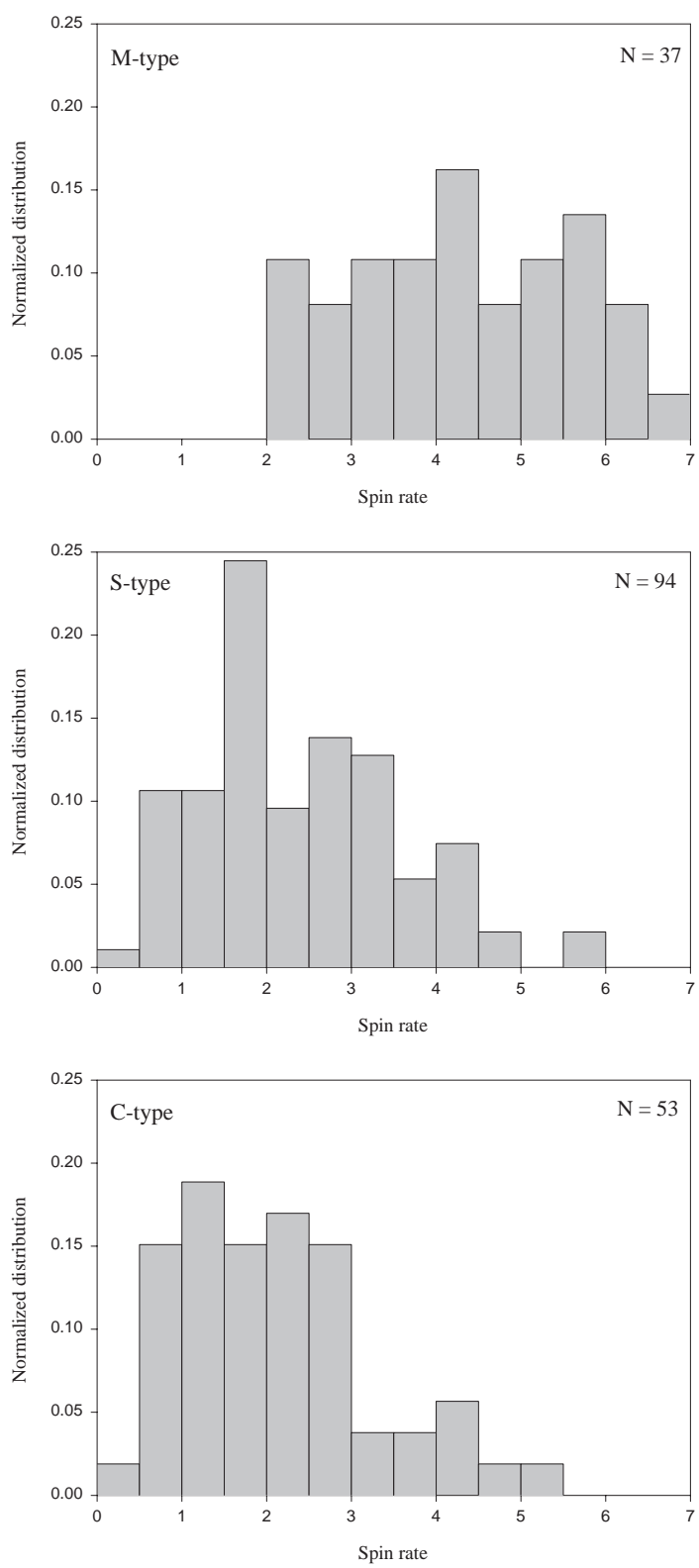

Fig. 14. Spin rate distributions for asteroids of taxonomic types $\mathrm{M}, \mathrm{S}$ and $\mathrm{C}$

92 Undina was first classified as C by Zellner \& Bowell (1979) because of a small value of the minimum polarization $(0.77 \%)$ and a large $U-B$ colour compared to other M-type objects. Tholen (1989) classified it as $X$ while the $u-v$ colour index of the asteroid differed too much from typical values. Barucci et al. (1987) and Tedesco et al. (1989) classified it as M. Jones et al. (1990) and later Rivkin et al. (1995) found the $3 \mu \mathrm{m}$ absorption band in spectra which indicate the presence of hydrated minerals 
on the asteroids surface and is therefore inconsistent with a metallic composition.

97 Klotho has quite typical classification parameters compared with the whole population of M-type asteroids. Radar observations of 97 Klotho failed to show any evidence of a metallic surface composition (Ostro et al. 1985). Thus, 97 Klotho should not be considered as a metal-rich body.

224 Oceana was classified as $\mathrm{M}$ based on its colours $U-$ $B=0.20 \mathrm{mag}$ and $B-V=0.75 \mathrm{mag}$ (Bowell et al. 1979). Both colours are at the border for the M-type population (see Fig. 13). Additional data are needed to check the classification.

322 Phaeo was classified as $X$ by Tholen (1989) and as M by Barucci et al. (1987) and Tedesco et al. (1989) based on its IRAS albedo of 0.088 (Tedesco et al. 1992). The albedo is one of the lowest among the M-type population. On the other hand the value of the $v-z$ colour (Zellner et al. 1985) is the highest among the EMP classes.

498 Tokio was classified as $U$ because of the large $U-B=$ $0.42 \mathrm{mag}$ (Bowell et al. 1979). Later it was classified as M by Tholen (1989) and as D3 by Barucci et al. (1987). Fitzsimmons et al. (1994) determined a spectral slope $S^{\prime}=$ $8.8 \%$ which is quite typical for asteroids of taxonomic type D (Dahlgren \& Lagerkvist 1995). Since 498 Tokyo has an albedo of only 0.07 (Tedesco et al. 1992) we conclude that the correct taxonomic type for this asteroid must be D.

1210 Morosovia was classified as SM by Bowell et al. (1979) because of the large $B-V=0.83 \mathrm{mag}$ which is closer to the mean value for the S-type population (0.86 mag) than to the mean value of $B-V(0.70 \mathrm{mag})$ for the M-type asteroids (Belskaya \& Lagerkvist 1996). Tholen (1989) classified the asteroid as MU since the ECAS data were noisy. The asteroid 1210 Morosovia is a member of the Eos family. Most probably it is of taxonomic type $\mathrm{S}$ since all other classified asteroids in the Eos family are of this taxonomic type (Tholen 1984).

The main conclusion of the discussion above is that these six asteroids cannot be considered to be members of the M-type population.

\subsection{Spin rates of asteroids of different taxonomic types}

The increase of the data set of M-type asteroids with known rotation periods, and the exclusion of six asteroids previously considered to be belonging to the M-type population, justifies a new comparison of the spin rates between the M, S and C asteroiods.

For comparison we chose asteroids with diameters larger than $70 \mathrm{~km}$ for which the statistics is almost complete for M-type asteroids. Considered asteroids of C- and S-type have semi-major axes between 2.3 and $3.2 \mathrm{AU}$ which is the same range as for M-asteroids. However, asteroids of taxonomic types $\mathrm{C}$ and $\mathrm{S}$ are not completely sampled down to this diameter regarding rotation periods. In practice this means that available rotational data for $\mathrm{C}$ and $\mathrm{S}$ asteroids down to this diameter is overrepresented by asteroids with short rotation periods.

In Fig. 14 we present the histograms for the rotation periods of the M, S and C type asteroids. The individual $\mathrm{M}$ asteroids shown above being untypical $\mathrm{M}$ asteroids have been excluded. It is clearly evident that $M$ type asteroids in general spin faster than asteroids of type $\mathrm{C}$ and $\mathrm{S}$. Considering the bias in the $\mathrm{C}$ and $\mathrm{S}$ population, favouring short periods, this difference is even greater than seen in Fig. 14. The distribution of the spin rates of $M$ type asteroids is also much flatter than for the other types. This is also verified by a Kolmogorov-Smirnov test (e.g. Press et al. 1989). The result of the test shows that the spin rate distribution for M-type asteroids is completely different from those of S- and C-type asteroids, while the later two types have large similarities of the observed spin rate distributions.

\section{Conclusions}

Most of the slow rotators found among the M-type asteroids have one or more of the classification parameters differing from the typical ones for the M-type population. For others radar observations, or spectra in the near infrared, show that they do not have the properties representative for the M-type population. The slow rotators dicussed above should therefore probably not be classified as type M. The enlarged data of spin rates of M-type asteroids confirms and strengthens the conclusion that asteroids of taxonomic type $\mathrm{M}$ spin faster than those of similar sizes of types $\mathrm{S}$ and $\mathrm{C}$.

Acknowledgements. We thank E. Braatz and Yu. Krugly for their help with the observations. The Kharkiv team was partly supported by the Ukrainian State Foundation of Fundamental Research (grant N 2.4/314). I. Belskaya is grateful to Uppsala University for financing her visit at the Uppsala observatory. J. Piironen wishes to thank NorFA foundation for financial support.

\section{References}

Barucci M.A., Capria M.T., Coradini A., Fulchignoni M., 1987, Icarus 72,304 
Belskaya I.N., Kiselev N.N., Lupishko D.F., Chernova G.P., 1991, Kinem. Fiz. Nebesn. Tel. 7, No. 6, 11 (in Russian)

Belskaya I.N., Lagerkvist C.-I., 1996, Planet. Space Sci. 44, 783

Binzel R.P., Farinella P., Zappala V., Cellino A., 1989, "in Asteroids II", Binzel R.P. Gehrels T., Matthews M. (eds.). Univ. of Ariz. Press, Tucson, p. 148

Bowell E., Gehrels T., Zellner B., 1979, in: Asteroids, Gehrels T. (ed.). Univ. of Ariz. Press, p. 1108

Dahlgren M., Lagerkvist C.-I., 1995, A\&A 302, 907

Dermott S.F., Murray C.D., 1982, Nat 296, 418

Dotto E., Barucci M.A., Fulchignoni M., Di Martino M., Rotundi A., Di Paolantinio A., 1992, A\&AS 95, 195

Fitzsimmons A., Dahlgren M., Lagerkvist C.-I., Magnusson P., Williams I.P., 1994, A\&A 282, 634

Harris A.W., Burns J.A., 1979, Icarus 40, 115

Harris A.W., Young J.W., 1979, Icarus 38, 100

Harris A.W., Young J.W., 1983, Icarus 54, 59

Harris A.W., Young J.W., 1989, Icarus 81, 314

Harris A.W., Young J.W., Dockweiler T., Gibson J., Poutanen M., Bowell E., 1992, Icarus 95, 115

Jones T.D., Lebofsky L.A., Lewis J.S., Marley M.S., 1990, Icarus 88, 172

Lagerkvist C.-I., 1983, in: Highlights of Astronomy, West R.W. (ed.). D. Reidel Publ. Co., p. 371

Lagerkvist C.-I., Hahn G., Magnusson P., Rickman H., Hammarbäck G., 1985, in: Asteroids, Comets, Meteors II, Lagerkvist C.-I. Lindblad B.A., Lundstedt H., Rickman H. (eds.). Uppsala Universitet, Reprocentralen, HSC, Uppsala, p. 67

Lagerkvist C.-I., Magnusson P., Williams I.P., Buontempo M.E., Gibbs P., Morrison L.V., 1988, A\&AS 73, 395

Lagerkvist C.-I., Erikson A., Debehogne H., Festin L.,
Magnusson P., Mottola S., Oja T., de Angelis G., Belskaya I.N., Dahlgren M., Gonano-Beurer M., Lagerros J., Lumme K., Pohjolainen S., 1995, A\&AS 113, 115

Lagerkvist C.-I., Magnusson P., Belskaya I., Piironen J., Warell J., Dahlgren M., 1996, Asteroid Photometric Catalogue, fourth update, Uppsala Univ., Reprocentralen HSC, Uppsala

Ostro S.J., Cambell D.B., Shapiro I.I., 1985, Sci 229, 442

Press W.H., Flannery B.P., Teukolsky S.A., Vetterlingm W.T., 1989, Numerical recipes (FORTRAN). Cambridge Univ. Press

Rivkin A.S., Howell E.S, Britt D.T., Lebofsky L.A., Nolan M.C., Branston D.D, 1995, Icarus 117, 90

Tedesco E.F., Williams J.G., Matson D.L., Veeder G.J., Gradie J.C., Lebofsky L.A., 1989, AJ 97, 580

Tedesco E.F., 1989, "Asteroid magnitudes, $U B V$ colors, and IRAS albedos and diameters, in Asteroids II, Binzel R.P. Gehrels T., Matthews M. (eds.). Univ. of Ariz. Press, Tucson, p. 1090

Tedesco E.F., Veeder G.J., Flower J.W., Chillemi J.R., 1992, The IRAS Minor Planet Survey Data Base, National Space Science Data Center, Greenbelt, Maryland

Tholen D.J., 1984, "Asteroid taxonomy from cluster analysis of photometry", Ph.D. Thesis Univ. of Arizona

Tholen D.J., 1989, "Asteroid taxonomic classification", in Asteroids II, Binzel R.P. Gehrels T., Matthews M. (eds.). Univ. of Ariz. Press, Tucson, p. 1139

Zellner B., Bowell, E., 1979, in "Asteroid compositional types and their distributions", in Comets, Asteroids, Meteorites, Delsemme A.H. (ed.). The University of Toledo, p. 185 Zellner B., Tholen D.J., Tedesco E.F., 1985, Icarus 61, 355 